\title{
Session D
}

\section{Atmospheric inhomogeneities}


N. E. Piskunov, W. W. Weiss, D. F. Gray, eds.

\title{
A New View of the Magnetic Sun
}

\author{
A. Title \\ Stanford-Lockheed Institute for Space Research, Palo Alto, CA 94304
}

\begin{abstract}
.
Developments in instrumentation, numerical simulations, and theory are rapidly changing our view of solar magnetism. There are now observations that show magnetic field emerging on all convective scales. The emergence rate replaces the quiet Sun flux in less than 12 hours and even active region and sunspot fields are replaced in less than a month. There is evidence for local dynamo action suggesting that a bottom to a convection zone is not required for stellar magnetic activity. It is now recognized that $3 \mathrm{D}$ magnetic reconnection is fundamentally different from $2 \mathrm{D}$. Time sequences of the one arc second spatial resolution TRACE images show that the temperature and density structure of the corona changes as fast as radiation and conduction allow. Because adjacent loops are observed in a range of temperatures that span at least 30000 to $2500000 \mathrm{~K}$, there is a inter mixture of temperatures regimes throughout the corona. Consequently, there is no line of sight through the corona that can be characterized by a single temperature and density. It would be surprising if other stars or other astrophysical systems with magnetic fields were simpler than the solar atmosphere.
\end{abstract}

\section{Introduction}

Observations in space by $\mathrm{YOHKOH}, \mathrm{SOHO}$, and TRACE and on the ground by the new observatories using digital detectors, new instruments, adaptive optics, and post processing have provided data with significantly higher measurement precision, higher spatial and temporal resolution, and more complete spectral and temporal coverage of the Sun's magnetic activity and its effects. These measurements now extend from near the solar core through its extended outer atmosphere. The observations have spurred theory and modeling toward developing insights into the global and local dynamo processes, the nature of magnetic reconnection, and heating mechanisms for the chromosphere, transition region, and corona. Because there are many new ideas and old ideas die hard it is useful to review briefly the history of magnetic field observations before the 1990's before turning to the discoveries and implications of recent observations and models.

Many of the subjects discussed here are much better visualized with movies than single images. Many movies can be found at http://www.lmsal.com. The presentation of this paper used a DVD - The Sun-Earth Connection. Copies are available. 


\section{A brief history}

In the first decade of the 20th century George Ellery Hale discovered, by direct observations of the Zeeman splitting of spectral lines, that sunspots have magnetic fields of about 2000 Gauss. Forty years later, the Babcocks built a photoelectric magnetograph and measured polar fields of less than 5 Gauss. Howard and Bumba measured a similar strength in unipolar regions that covered a large fraction of the solar surface. These unipolar regions are the remnant fields that trail away from the positions of decaying spots and plage. In the 1960's, as magnetographs improved both in magnetic sensitivity and spatial resolution, more and more weak fields were discovered. All of the new photoelectric magnetographs traded sensitivity for dynamic range and as a result saturated at about 5 Gauss. Then in 1965 Sheeley, using the dual beam photographic spectroheliograph developed by Leighton, showed that much of the field outside of sunspots was 300 Gauss or more.

Shortly thereafter, Stenflo and Frazer argued, based on line ratio arguments, that more than $90 \%$ of the field on the Sun was on the order of a kiloGauss. These line ratio measurements were repeated for many areas of the Sun and always returned the result that virtually all of the solar fields were about a kiloGauss. Tarbell and Title took spectra-spectroheliograms of large areas of the Sun. They measured Zeeman splittings from spectra with one arc second resolution and found that the field strength in the mid-photosphere was $1100+/-$ 100 Gauss where flux could be detected, but could not rule out the presence of fields less than 100 Gauss or weak fields mixed with strong fields. Spruit showed how kiloGauss fields, which have an energy greater than the equipartition, could occur by the mechanism of convective collapse and predicted that the strong fields would be contained in the intergranular lanes.

In parallel with the magnetograph measurements, there were new theories of the solar dynamo. Babcock suggested that the sunspot fields were generated beneath the surface, but not at the bottom of the convection zone, by winding of the fields by the solar differential rotation. The Babcock processes converted an initial poloidal field to a toroidal field. Leighton amplified on this idea. $\mathrm{He}$ created an $\alpha-\omega$ dynamo using the observed tilt of the sunspot bipoles for the $\alpha$ component. Then a combination of dispersal generated by supergranules and differential rotation spread the decaying sunspot fields over the surface. Because the flux of the leading spot cancelled more readily across the equator than that of the following, the polar field in each hemisphere developed the polarity opposite to that of the leading spots. This created the reversal of the polar fields required for the new cycle. In the polar regions the field was subducted and subsurface differential rotation generated new toroidal field that finally emerged as the next cycle sunspots.

Leighton's model did not provide a mechanism for subduction of the polar field nor did it suggest where below the surface differential rotation amplified the field, but it did produce many of the features observed by the magnetographs. Leighton's graduate student, Jim Mosher, soon showed that the spreading of the field by supergranulation, for which Leighton had assigned a diffusion constant in his models, occurred at a rate a factor of five slower than used in the models. As a result, the cycle time created by the models was increased by more than an order of magnitude. Mosher suggested that the proper cycle duration would 
occur if there existed a meridional flow between 10 and $20 \mathrm{~m} / \mathrm{s}$. Duval soon, thereafter, measured a poleward flow of $20 \mathrm{~m} / \mathrm{s}$.

Sheeley and his collaborators have developed a series of models of the surface distribution of magnetic fields by diffusion, differential rotation, and meridional flow. These calculations have been remarkably successful predicting the evolution the large-scale distribution of the flux on the solar surface after the observed emergence of active regions. In addition, Sheeley has shown that the top ten percent of the active region emergences causes most of the features of the large-scale surface distribution.

The development of the Kitt Peak 512 channel magnetograph by Livingston and Harvey led to the discovery of small-scale fields nearly everywhere on the solar surface. While this was exciting, it was regarded by most as a kind of magnetic noise that had no effect on the large-scale distribution of magnetic fields. Interest in the small-scale fields increased when the images produced by Skylab X-ray telescope revealed that compact bright features, X-ray bright points, occurred essentially everywhere on the solar surface. Nearly simultaneous with the X-ray bright point observations was the discover by Martin and Harvey of small bipoles, ephemeral region, less than one hundredth the flux of active regions, that emerged all over the solar surface and that lived less than a day. Initially, the X-ray bright points were thought to be caused by the interaction of the rising ephemeral regions with the overlying coronal fields. However, Karen Harvey soon showed that about two thirds of the X-ray bright points were instead associated with cancellation of magnetic flux.

Karen Harvey extensively studied the ephemeral component of the smallscale fields. She defined ephemeral regions as short-lived bipoles with a separation between poles of 2.5 degrees or less. Her measurements showed that the total flux of all ephemeral regions on the Sun varied by only a factor of two over solar cycle rather than the factor of eight exhibited by the active region fields.

In the same period Smithson and Mosher developed a video magnetograph system that could take 15 magnetograms a second and add thousands of individual magnetograms in real-time. This new technique achieved high signal-to-noise data in a relatively short period. Using the video magnetograph movies, Martin showed the existence of yet another smaller component of the flux, regions that appeared inside supergranules, the internetwork flux. Her data strongly suggested that the internetwork fields were weak, 500 Gauss or less. Measurements of ephemeral regions and especially internetwork fields are difficult because of variable seeing and the transient nature of the fields. For this reason, groundbased measurements often provided conflicting results that were hard to properly interpret.

In the last decade of the previous millennium, the new Swedish VVT and the new HAO Advanced Stokes Polarimeter began operation. In addition, new numerical simulations were making predictions about solar granulation that were quickly validated by the new observations in the continuum made in space by the SOUP instrument and on the ground by the SVTT. Observations in the G band ( $\mathrm{CH}$ band head at $4308 \AA$ ) showed that the small-scale magnetic field was largely contained in the intergranular lanes as predicted by modeling. Stokes polarimetry in the IR began to measure weak field in the quiet Sun. 

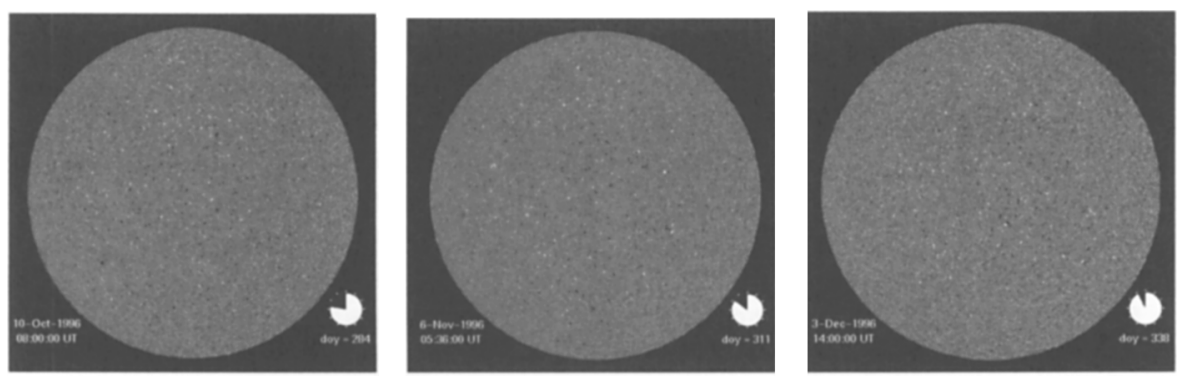

Figure 1. MDI full disk magnetograms for three successive rotations in the 1996 solar minimum.

\section{The Magnetic Carpet}

The Michelson Doppler Imager on SOHO makes measurements of the line of sight magnetic field at least once every 96 minutes. First turned on scientifically in solar minimum, it immediately revealed mixed polarity magnetic field dynamically evolving everywhere on solar surface. This mixed polarity persisted in spite of the fact that no new active regions appeared for months at a time. Figure 1 shows three magnetograms of the Sun at three successive rotations. There are no active regions in this period yet the Sun is always covered with mixed polarity fields. Kinematic simulations by Simon, Weiss, and Title indicated that the supergranulation flow would remove the mixed polarity component in a few turn over times -24 to 100 hours. They suggested that the observed mixed polarity field requires a constant emergence of bipoles, which emerged on a spatial scale less than supergranulation. Hagenaar developed sophisticated bipole identification algorithms and showed, using as her data base long sequences of MDI magnetograms with a one-minute cadence, that in fact the quiet sun magnetic network was maintained by the emergence of new bipoles at a rate sufficient to renew it in less than 12 hours.

New bipole identification was difficult with groundbased data because the ephemeral class bipoles emerge fast and then quickly mix with the existing mixed polarity fields in the supergranulation boundaries. The bipoles appear to be the ends of loops that rise through the photosphere. As a loop rises the footpoints initially separate at about $4 \mathrm{~km} / \mathrm{s}$ to a separation of about $7500 \mathrm{~km}$ - the diameter of the loop. Thereafter, the supergranulation flow separates the footpoints further with a speed of $1 \mathrm{~km} / \mathrm{s}$. The new bipoles merge completely with the existing network fields in less than four 4 hours. The daily full Sun Kitt Peak magnetograms were not the optimal data set for determining the rate of ephemeral region emergence.

Ephemeral regions seldom disappear by retracting. Rather, they cancel, with some previously existing field in a network boundary. The process of emergence, fragmentation, and disappearance can be described in the language of chemical kinetics (see Schrijver et al.). There are many consequences of the mixed polarity fields. First, neglect of the mixed polarity component of the field seriously underestimates the amount of magnetic field emerging from the solar surface. At arc minute resolution unipolar regions have a net flux of 0.1 to 0.5 


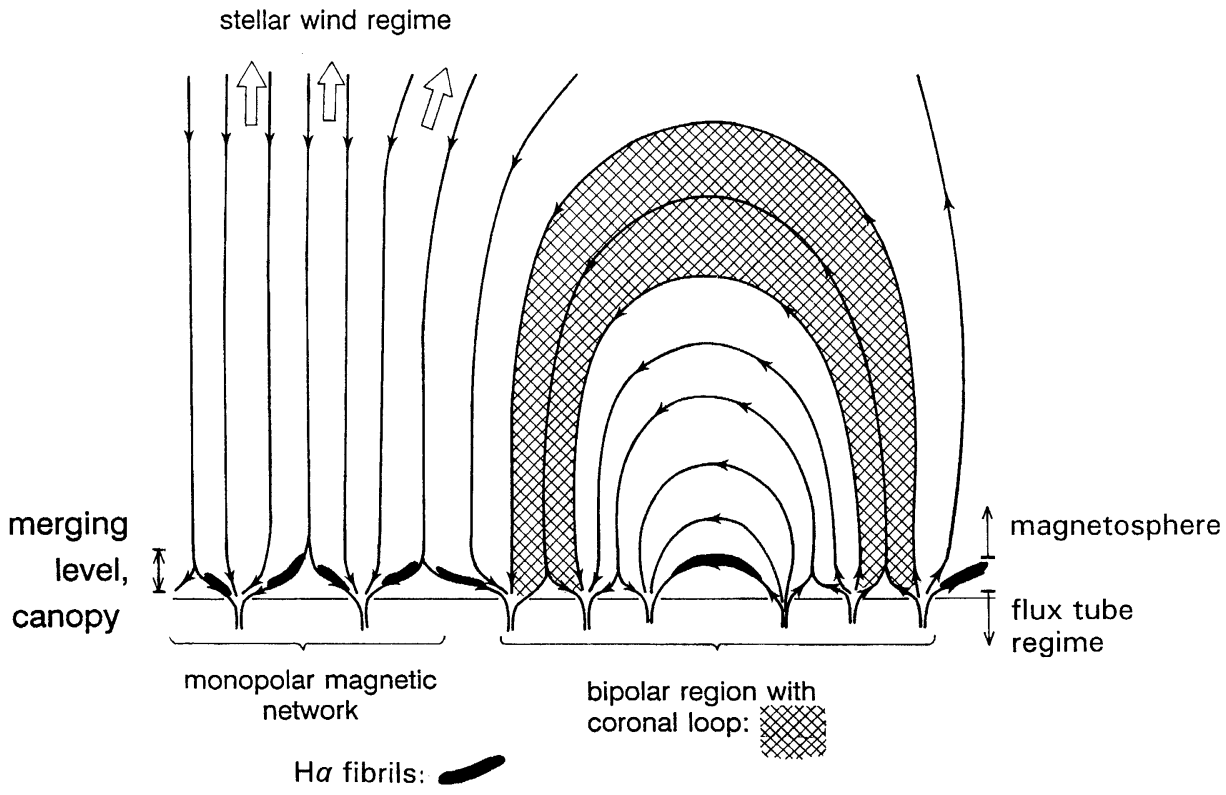

Figure 2. The standard cartoon representing the magnetic field over a unipolar magnetic region of quiet Sun.

Gauss just as predicted by the surface diffusion models. Observed at the 3 arc second resolution of summed and smoothed MDI magnetograms, the average absolute value of the flux in quiet Sun is in the range of 4 to 10 Gauss. That is, there is between 10 and 100 times more magnetic field on the surface than was previously estimated from models of surface spreading, which do not contain the ephemeral component of the field.

The existence of the mixed polarity fields fundamentally changes the picture of how fields extend above the photosphere. The standard cartoon shows unipolar flux emerging from the boundaries of supergranules (see figure 2 for an example) then spreading laterally to uniformly fill the corona with field of a single sign. The rapid spreading produces a canopy of nearly horizontal fields just above the photosphere. The canopy fields point from the supergranule boundaries to the cell centers. Unfortunately, this picture ignores at least $90 \%$ of the flux extending into the chromosphere and a smaller fraction of the field reaching the corona, In addition, it ignores the very complex tangle of loops that span a range of lengths produced by the mixed fields. A better representation of the magnetic field above the solar surface is given by a potential field extrapolation from an MDI magnetogram (see figure 3).

It is generally agreed that heating of the corona and the transition region is related to processes occurring in magnetic fields, although the mechanisms that cause the heating, or even where the heating occurs continues to be a subject of considerable discussion. This has been caused in large part by the observations of TRACE that have shown that the bright coronal structures evolve so rapidly and are so dynamic that they can not be explained by quasi-static or quasi- 


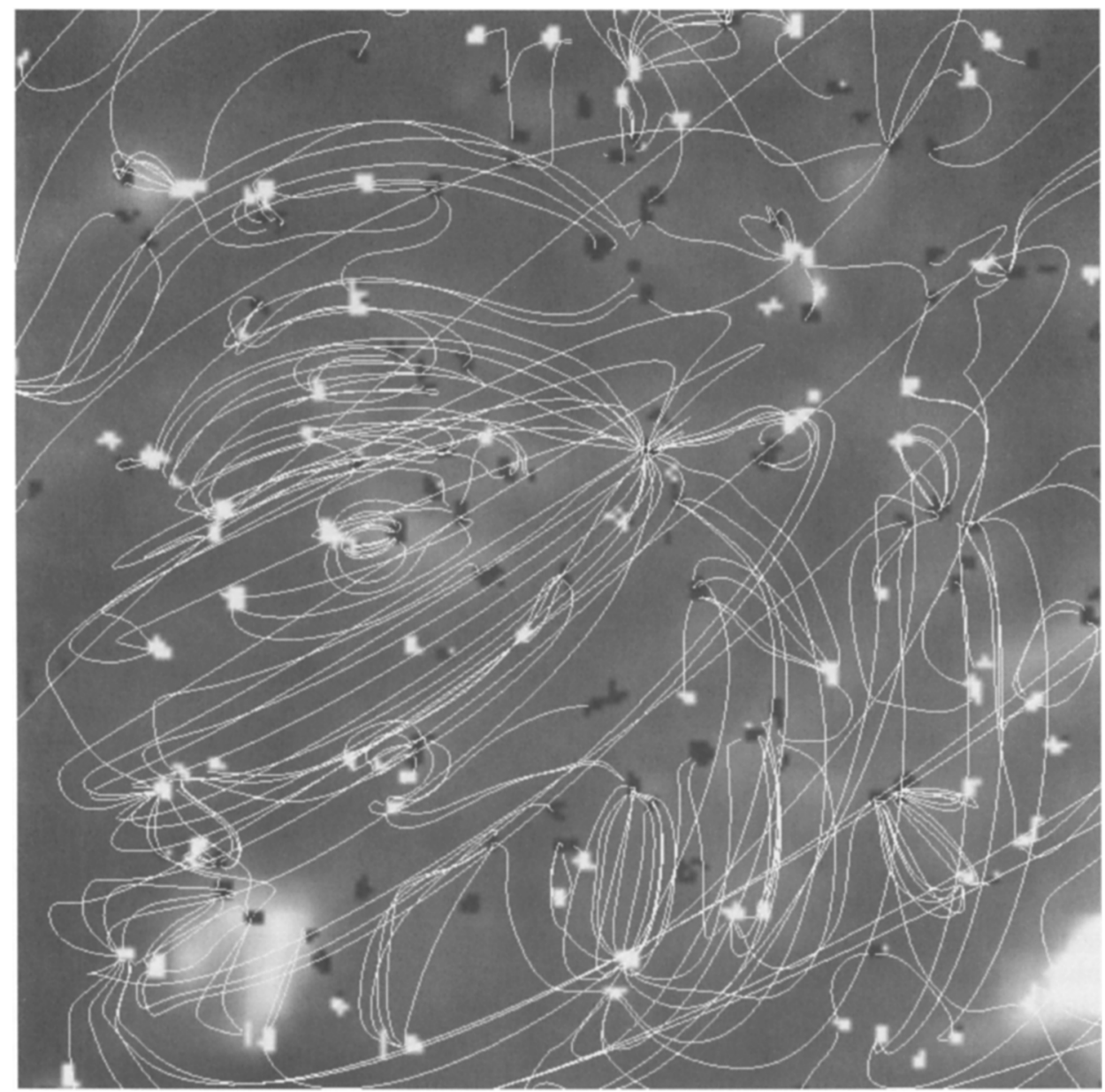

Figure 3. A potential field extrapolation from a MDI magnetogram in quiet Sun.

equilibrium models. However, it was surprising to most that the locations of foot points of these bright coronal loops were not coincident with the locations magnetic fields indicated by bright regions in the Calcium K line and G-band bright points. Careful cross-correlations between loop footpoint maps and $\mathrm{K}$ and G band images by De Pontieu et al. yielded near zero correlation at the one arc second resolution scale of the TRACE images. At a resolution of 5 arc seconds the cross correlation rises toward 0.8 , which shows that loops do end, as is expected, in areas of surface magnetic flux. Much of the surprise must be associated with the familiar cartoon shown in figure 2, which shows field lines rising in expanding bundles from the magnetic network. With this picture in mind it is difficult to understand the observations.

A major consequence of the rapid replacement time of the magnetic carpet is that no field lines can stay connected to the same surface magnetic flux con- 


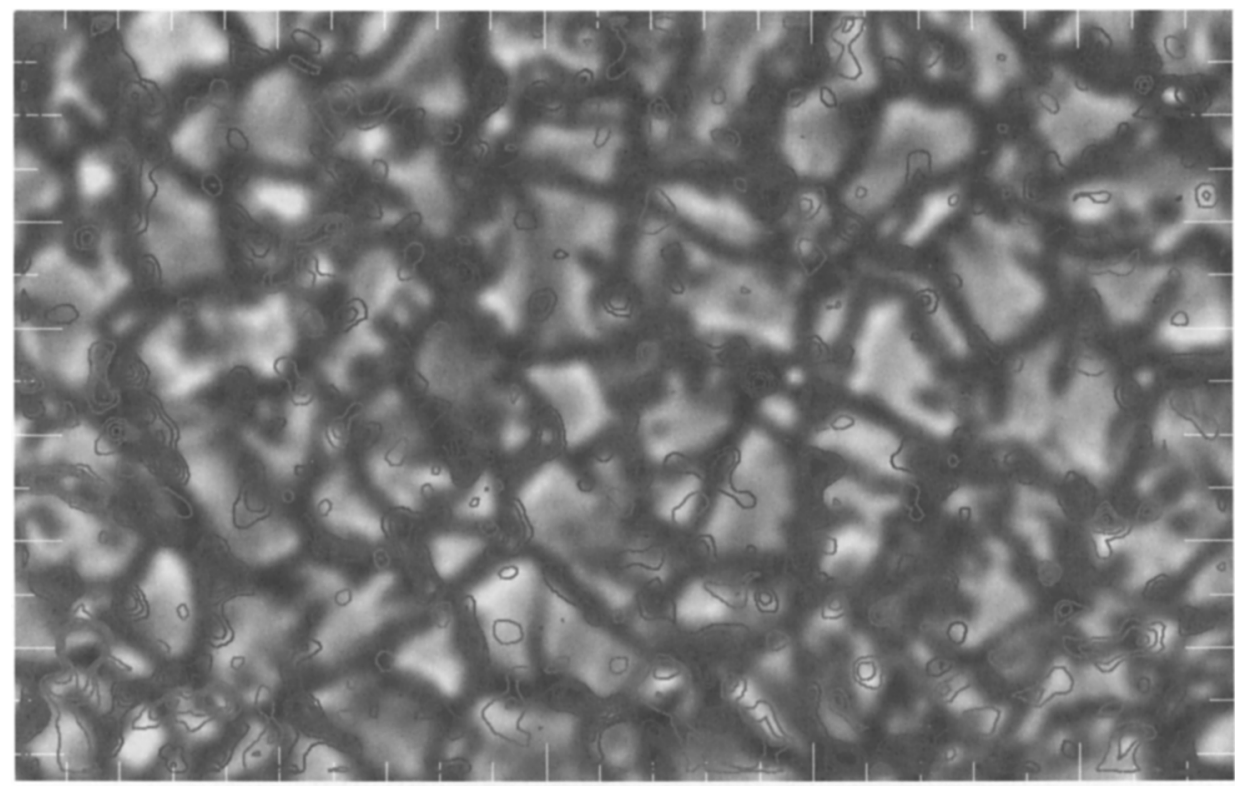

Figure 4. A high resolution high sensitivity magnetogram showing magnetic field in the granulation boundaries (courtesy of Cerdena, Kneer, and Almeida).

centration in the photosphere for much more than 12 hours in quiet Sun. Even in active regions the maximum connection time is a few weeks. This is a stronger statement than that the ends of all magnetic flux concentrations are constantly being fragmented and moved by the solar granulation. Before the rapid replacement of the surface magnetic field was understood, observations had shown that magnetic flux was largely contained in the intergranular lanes. Because granules themselves changed on a five-minute time scale, this implied that the ends of loops wandered about the photosphere. Observations of G-band bright points, which are surrogates for magnetic flux locations, showed them fragmenting and merging in the granules boundaries as the flow field evolved. Berger and Title had even called into question of whether the construct of magnetic flux tubes was useful.

It is now clear that ephemeral bipoles are different from the majority of the internetwork flux concentrations that are weak with field strengths that may be as small as 50 Gauss. Lites using the HAO Stokes polarimeter has reported weak horizontal fields scattered all over the solar surface that may be different from the "standard internetwork" structures. In addition, De Pontieu and Scharmer have reported on occasionally seeing the emergence of small bipoles on the scale of granulation with an observed lifetime on the order of ten minutes. Very recently high-resolution magnetograms made by Cerdena have revealed kiloGauss magnetic field in essentially all granulation boundaries in very quiet Sun (see figure 4). The body of magnetic observations suggests that field is emerging onto the surfaces on all scales of convection. 


\section{The Magnetic Carpet and the Solar Dynamos}

The observations of the magnetic carpet and advances in computer technology have renewed interest in developing numerical simulations that can reproduce some of the features of the solar magnetic field observations. Cattaneo, using incompressible simulations, has demonstrated local dynamo action. His models start with an initial weak random seed field and evolve to a steady state where $25 \%$ of the energy originally in convection is converted into magnetic energy. The fields are contained in the boundaries of the convection flows. Shown in figure 5 is a magnetic field map from a time step in the simulation. The magnetic map has long strings of mixed polarity field and mixed spirals of magnetic field that have not yet been observed on the Sun. When the images are blurred the thin lines of field vanish and the swirls either disappear or become round flux concentrations of a single sign. Movies of the simulated magnetic field evolution, however, look different than MDI magnetic movies. The main reason for this is that the solar ephemeral regions appear in pairs, while the blurred simulation has concentrations that randomly rise above and then fall below a detection level.

Nordlund and Stein (NS) have also recently added magnetic interactions to their near surface simulations. The NS simulations are more realistic than those of Cattaneo in that they are compressible, use the solar pressure variations, and include radiation; but dynamo action does not occur in their simulations. The reason for this is the lack of mixing of the up and downflows that occurs because of their penetrating lower boundary condition, whereas Cattaneo's simulations have a solid lower boundary that causes turbulent mixing at that boundary. Nevertheless, when NS insert uniform sheets of horizontal magnetic fields into the bottom of their simulation volume the surface distribution of the magnetic field is strikingly similar to that which occurs in the incompressible simulation. When their maps are blurred to 0.2 arc second resolution, they are similar to observations (see figure 6 for the original and blurred magnetogram pairs).

At present, computers are not adequate to approximately model the entire convection zone. Even without including magnetic fields it is not yet possible to reproduce the internal rotation profile observed by helioseismology. It is widely, but by no means universally, agreed that toroidal magnetic field is amplified just beneath the convection zone to strength of about $10^{5}$ Gauss. The flux then rises through the convection zone and about a month later appears on the solar surface. When the flux emerges on the surface, the leading polarity has an average tilt toward the equator caused by the Coriolis forces acting on the tube during its travel to the surface. The simulations that model rising flux tubes neglect turbulence in detail and have assume that the emerging flux tubes are twisted to prevent instabilities that would otherwise disrupt them during their rise through the convection zone.

Further, there is really no good mechanism for subducting flux to below the convection zone. Models by Nordlund and Brummel have shown that the near surface fields can be carried downward by the narrow downflows in the granule boundaries, but these models appear to deposit fields throughout the convection zone. Dorch has created dynamo models that have a distributed but concentrated interior field. In short, there is no good model for the solar dynamo responsible for the sunspots and active regions. Nevertheless, many accept the 


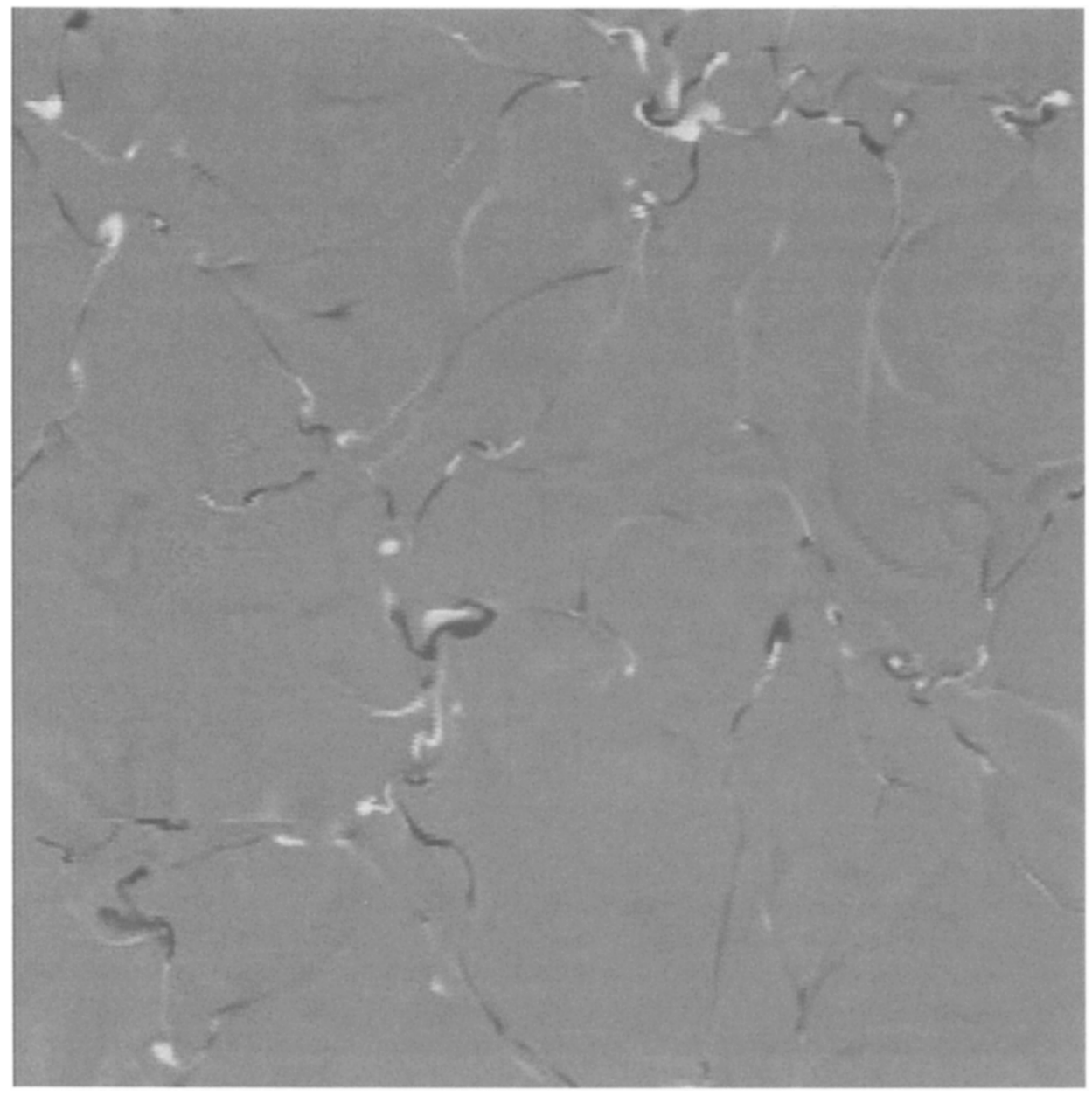

Figure 5. A magnetic map from an incompressible simulation that exhibits local dynamo action (courtesy Cattaneo). 


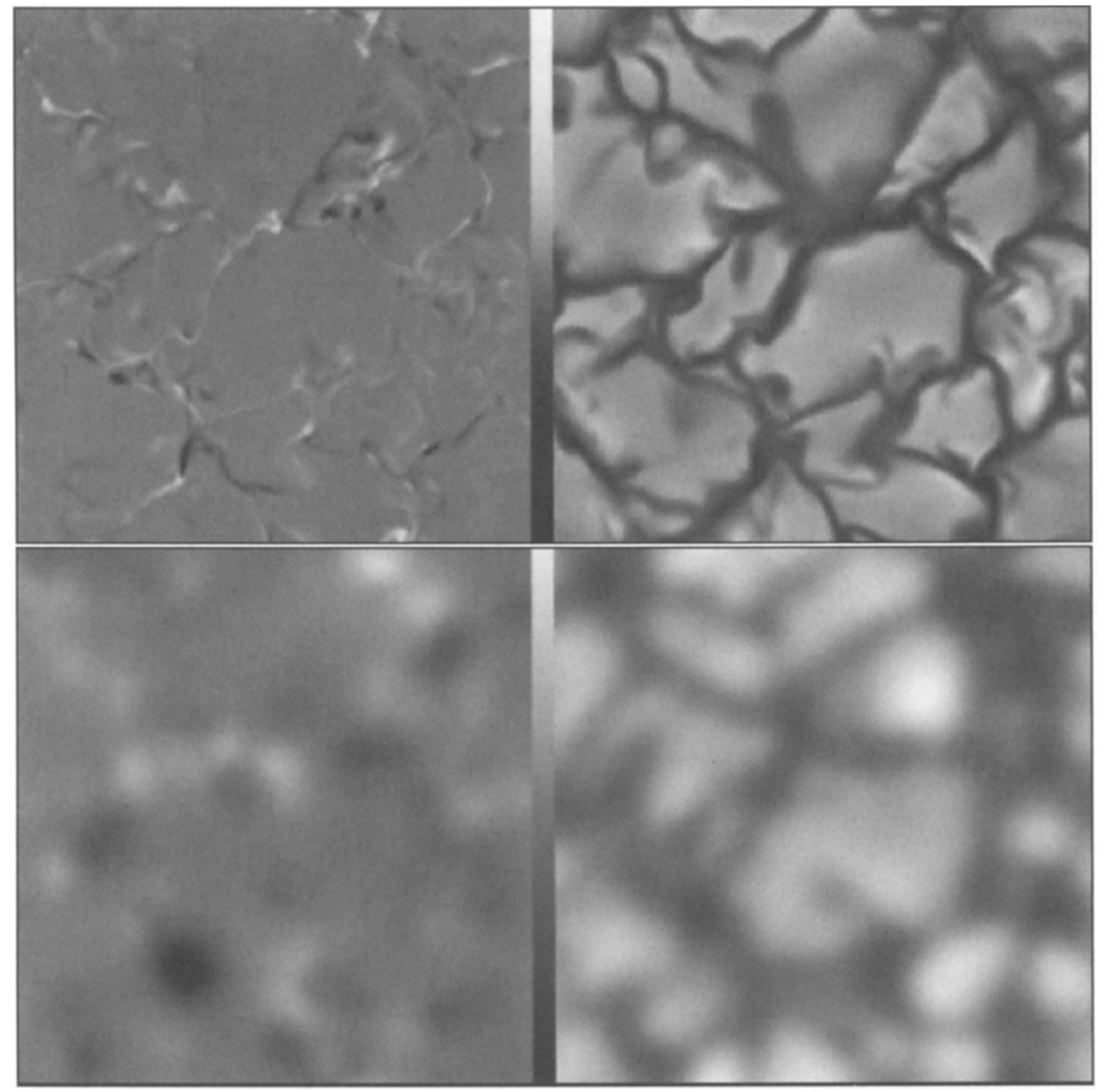

Figure 6. A magnetic map and corresponding intensity map from a compressible simulation (top) the same data but blurred to 0.2 arc seconds to simulate observational data (bottom, courtesy Nordlund and Stein). 
Babcock-Leighton idea. However, the evidence that a lower boundary to the convection zone or that differential rotation is required for dynamo action does not exist.

As mentioned earlier Harvey found that the total flux in ephemeral region only varies by about a factor of two over the solar cycle. However, Harvey only classified bipoles as ephemeral regions on the basis of their size. Hagenaar, using MDI magnetogram sequences, has been able to create distribution functions of the emergent quiet Sun flux as a function of total flux for all phases of the solar cycle. Bipoles with flux below $2 \times 10^{19} \mathrm{Mx}$ vary in antiphase with the cycle and are 1.5 times more prevalent at minimum than maximum. The result indicates that the smaller ephemeral regions are only weakly, if at all, coupled, with the solar cycle dynamo and suggests, but certainly does not establish, that dynamo action is occurring in the convection zone. Schussler and Schmidt have made models that indicate the variations observed in the solar cycle duration and amplitude can be caused by coupling between deep and local dynamos.

\section{The Magnetic Carpet and Energy Release}

The field in the quiet Sun is in a statistically fluctuating steady state: as much magnetic field is disappearing as appearing. If we estimate the magnetic energy as the magnetic energy in the emerging loop and use the area of the typical cell as the area factor and the replacement time as the rate, then the energy dissipation rate, $\mathrm{E}_{D}\left(\mathrm{ergs} / \mathrm{cm}^{2} / \mathrm{sec}\right)$ is

$$
E_{D}=B F /\left(8 \pi^{2} R T\right)
$$

where $B$ is the magnetic field strength (Gauss), $F$ is the total flux $\left(\mathrm{Mx} / \mathrm{cm}^{2}\right), \mathrm{R}$ is the cell radius $(\mathrm{cm})$, and $\mathrm{T}$ is the flux replacement time $(\mathrm{sec})$. Alternatively, this equation can be considered as an estimate of the work done by the flow field to move the magnetic field to where in can cancel with an opposite polarity flux element.

For ephemeral regions the average properties are $\mathrm{F}=8 \times 10^{18} \mathrm{Mx}, \mathrm{B}=1200$ Gauss, $\mathrm{R}=10^{9} \mathrm{~cm}$, and $\mathrm{T}=36000$ seconds, which yields an average energy dissipation rate due to ephemeral regions of

$$
E_{D}(\text { network })=3.4 \times 10^{6} \mathrm{ergs} / \mathrm{cm}^{2} / \mathrm{sec} \text {. }
$$

For the internetwork fields $\mathrm{F}=1 \times 10^{17} \mathrm{Mx}, \mathrm{B}=50-200$ Gauss, $\mathrm{R}=10^{8} \mathrm{~cm}$, and $\mathrm{T}=3600$ seconds, which yields an average energy dissipation rate of

$$
E_{D}(\text { internetwork })=2-7.0 \times 10^{5} \mathrm{ergs} / \mathrm{cm}^{2} / \mathrm{sec} .
$$

The newly discovered field on the scale of granulation seems to have kiloGauss field strength. The preliminary estimates for the properties of these granule boundary fields are $\mathrm{F}=5 \times 10^{16} \mathrm{Mx}, \mathrm{B}=1200$ Gauss, $\mathrm{R}=5 \times 10^{7} \mathrm{~cm}$, and $\mathrm{T}=$ 300 seconds, which yields an energy dissipation rate of

$$
E_{D}(\text { granulation })=5 \times 10^{7} \text { ergs } / \mathrm{cm}^{2} / \text { sec } \text {. }
$$




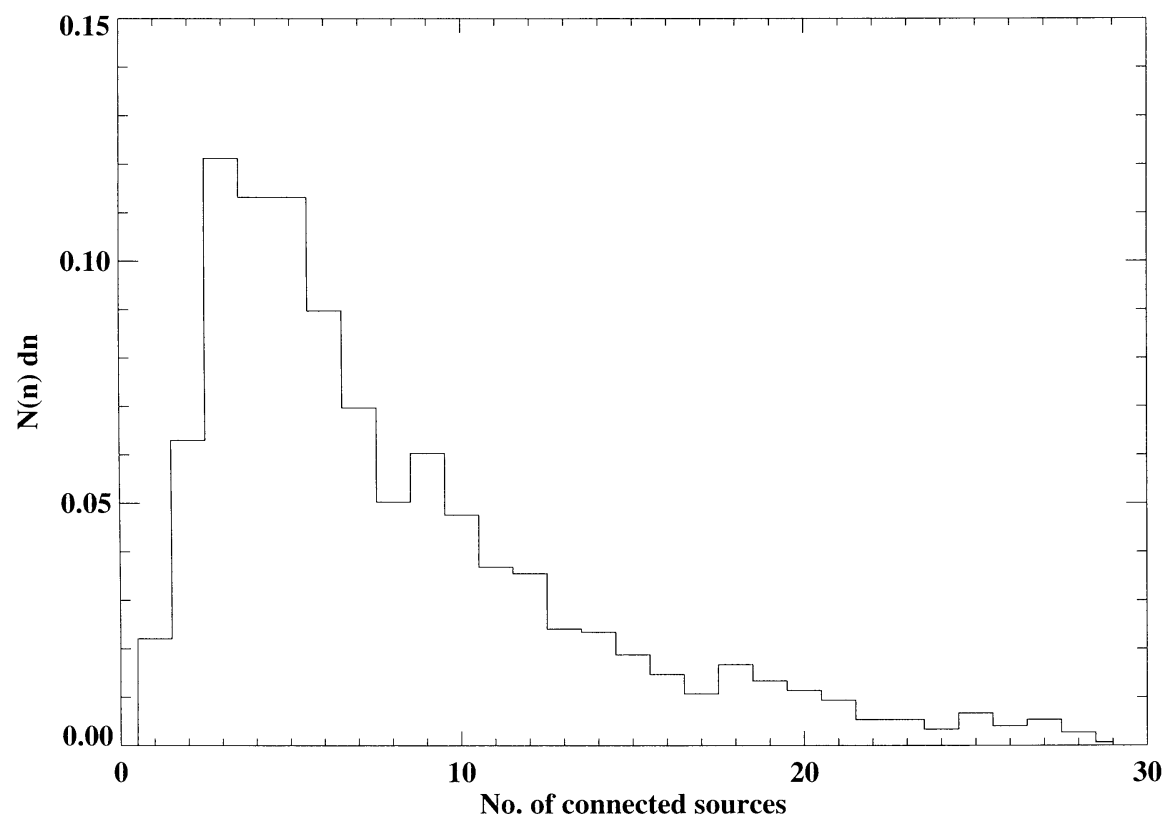

Figure 7. The distribution function of the number of sources to which a source in a random mixed polarity region is connected.

Each of these numbers is greater than the $10^{5} \mathrm{ergs} / \mathrm{cm}^{2} / \mathrm{sec}$ that is estimated to be required to heat the corona. However, it is critical to know how and where the energy is released. Since most of the magnetic field of a dipole is inside a height less than the separation of the charges, it might be expected that in quiet Sun most of the energy would be released within a few thousand kilometers from the surface, i.e. in the chromosphere and low corona.

Some fraction of quiet Sun field lines must be long as they connect between unipolar regions on the surface, but as discussed above only between 1 to 10 percent of the field lines in the carpet are in this category. However, in addition, the topological properties of mixed magnetic fields results in many longer connections than might be expected by the separations from the nearest neighbors of opposite polarity. Schrijver and Title have examined the properties of potential fields of mixed polarity magnetic regions with net zero flux. The magnetic charges were randomly distributed and had random strengths. As should be expected, it was found that most field lines from a magnetic charge connected with its four nearest neighbors of opposite polarity. But, because of the complex topologies generated, a significant fraction connected to other more distant sources. Shown in figure 7 is a plot of the distribution function of the number of other different charges an average charge is connected with. These potential field calculations show that about 40 percent of the field lines in a mixed polarity region would be connected sources with a separations as great as 5 to 6 times the average separation of the charges. Even for the flux on the scale of granulation 
there must be many field lines that extend 30 to 60 thousand kilometers, and thus extend well into the corona.

How energy is released in a mixed-polarity environment is beginning to be investigated via numerical simulations and theory. 3D magnetic reconnection is fundamentally different from $2 \mathrm{D}$, which requires a new lexicon of separators, separaterices, null points, spines, and fans. A discussion of $3 \mathrm{D}$ reconnection cannot be included here, but it appears that proper understanding will result in many insights into both slow and rapid release energy release processes in the solar atmosphere. Many of the initially mysterious field evolutions seen in the TRACE data become understandable when the magnetic topology of the region is estimated. From modeling of potential field configurations it is clear that the effect of motions of magnetic charges in a field pattern or the insertion of new of magnetic field into an existing configuration will differ significantly depending on where in the original configuration they occur. The reader is referred to papers by Priest, Longcope, and Demoulin for more information.

Movies made with the TRACE satellite have made the complexity of the corona impossible to avoid. The Sun's outer atmosphere is structured at the one arc second level observable by TRACE and it would be surprising if the fine structure ended there. The fine structure evolves as fast as conduction and radiation allow. Because adjacent loops are observed in a range of temperatures that span at least 30000 to $2500000^{\circ} \mathrm{K}$, there is a mixture of temperatures regimes throughout the corona. This complex topology means that there is no line of sight through the corona that can be characterized by a single temperature and density. It would be surprising if other stars or astrophysical systems with magnetic fields were simpler than the solar atmosphere. This means that no spectrum from a corona can be interpreted as coming from a volume with a single temperature and density. 